\title{
Circulating apelin, chemerin and omentin levels in patients with gestational diabetes mellitus: a systematic review and meta- analysis
}

Jianran Sun ${ }^{1 \dagger}$, Jiale Ren ${ }^{2 \dagger}$, Chunlin Zuo ${ }^{3 \dagger}$, Datong Deng ${ }^{3}$, Faming Pan ${ }^{4}$, Ruoping Chen ${ }^{1}$, Jie Zhu ${ }^{1}$, Chao Chen ${ }^{1}$ and Shandong $Y e^{1 *}$ (D)

\begin{abstract}
Background: The available data on the significance of circulating apelin, chemerin and omentin in women with gestational diabetes mellitus (GDM) are inconsistent. This analysis includes a systematic review of the evidence associating the serum concentrations of these adipokines with GDM.

Methods: Publications through December 2019 were retrieved from PubMed, Embase, the Cochrane Library, and Web of Science. Subgroup analysis and meta-regression were conducted to evaluate sources of heterogeneity.

Results: Analysis of 20 studies, including 1493 GDM patients and 1488 normal pregnant women did not find significant differences in circulating apelin and chemerin levels (apelin standardized mean difference $[S M D]=0.43,95 \%$ confidence interval $(\mathrm{Cl}):-0.40$ to $1.26, P=0.31$; chemerin $\mathrm{SMD}=0.77,95 \% \mathrm{Cl}-0.07$ to $1.61, P=0.07)$. Circulating omentin was significantly lower in women with GDM than in healthy controls (SMD $=-0.72,95 \% \mathrm{Cl}-1.26$ to -0.19 , $P=0.007$ ). Publication bias was not found; sensitivity analysis confirmed the robustness of the pooled results.
\end{abstract}

Conclusions: Circulating omentin was decreased in GDM patients, but apelin and chemerin levels were not changed. The results suggest that omentin has potential as a novel biomarker for the prediction and early diagnosis of GDM.

Keywords: Apelin, Chemerin, Omentin, Gestational diabetes mellitus, Meta-analysis

\section{Background}

Gestational diabetes mellitus (GDM) affects 5 to $20 \%$ of pregnant women, depending on the diagnostic criteria, population, and racial or ethnic group. According to the American Diabetes Association (ADA) criteria, GDM is defined as diabetes diagnosed in the second or third trimester of pregnancy that was not overt prior to gestation $[1,2]$. GDM increases the risk of hypoglycemia,

\footnotetext{
* Correspondence: shandongye@yeah.net

†Jianran Sun, Jiale Ren and Chunlin Zuo contributed equally to this work. 'Division of Life Science and Medicine, Department of Endocrinology, The First Affiliated Hospital of USTC (Anhui Provincial Hospital), University of Science and Technology of China, 17 Lujiang Road, Hefei 230001, China Full list of author information is available at the end of the article
}

hypocalcemia, and hyperbilirubinemia at birth, and the risk of developing glucose intolerance during childhood, adolescence, or adulthood [3, 4].

Understanding the pathogenesis of GDM is the key to preventing its development during pregnancy [5]. A progressive decline in insulin sensitivity and the development of $\beta$-cell dysfunction are believed to increase the risk of GDM [6, 7]. Recent evidence indicates that dysregulation of the secretion of adipokines, which are produced by adipose tissue, is involved in the development of insulin resistance (IR) during pregnancy [8].

Apelin, a bioactive peptide, was first identified in an extract of bovine stomach tissue. Both apelin and its 
receptor are expressed in various tissues, including the central nervous system, adipocytes, and the placenta [9]. Elsehmawy et al. [10] have described apelin as a novel adipokine that is produced and secreted by mature human adipocytes. Studies of serum or plasma apelin concentration in GDM patients by Baris et al. found lower circulating apelin in women with GDM than in controls; however, Emel et al. reported an increased apelin concentration in GDM patients [11, 12].

Chemerin, also called retinoic acid receptor responder protein 2 (RARRES2) or tazarotene-induced gene 2 protein (TIG2), is translated as an $18 \mathrm{kDa}$ inactive precursor protein and converted to the $16 \mathrm{kDa}$ active form by cleavage of the $\mathrm{C}$-terminus by an extracellular serine protease [13]. Chemerin was identified as an adipokine by Bozaoglu et al. who described its activity in regulating adipogenesis and adipocyte metabolism associated with metabolic syndrome [14]. Not all studies found a correlation between circulating chemerin and GDM. Yang et al. found that circulating chemerin was significantly elevated in women with GDM compared to controls, while Sadia et al. failed to find a significant association between chemerin concentration and GDM $[15,16]$.

Omentin, or intelectin-1, is an adipokine consisting of 313 amino acids primarily secreted from visceral adipose tissue, but it is also expressed in the heart, placenta, and ovaries [17]. Adipokines likely influence GDM; however, data on the roles of apelin, chemerin, and omentin in GDM are limited and inconsistent [18]. The aim of this study was to systematically evaluate the correlations between the serum levels of such adipokines and GDM.

\section{Materials and methods}

\section{Search strategy}

The meta-analysis was performed following the Preferred Reporting Items for Systematic Reviews and Meta-analysis (PRISMA) guidelines [19]. Studies published in English between January 1970 and December 2019 were retrieved from PubMed, Embase, the Cochrane Library, and the Web of Science using the text and MeSH terms "adipokines" and "gestational diabetes mellitus." The search terms included "apelin" or "apelin, AGTRL1 ligand, human" or "chemerin" or "chemerin proteins" or "omentin" or "intelectin, human" combined with "gestational diabetes mellitus" or "GDM". A full description of the search terms and strategy is available in the Supplementary material.

\section{Inclusion and exclusion criteria}

The inclusion criteria were as follow: a) Studies of any design, including cross-sectional, case-control, and clinical cohort studies; b) studies providing detailed data regarding serum or plasma apelin, chemerin, or omentin levels in patients with GDM and healthy controls; and c) studies in which the language was restricted to English.

The exclusion criteria were as follows: a) Studies that evaluated women with all types of diabetes mellitus (e.g., type 1 diabetes, type 2 diabetes, or specific types of diabetes due to other causes) and did not provide precise data on GDM; b) studies of fetal and/or placental tissues, such as cord blood an placental biopsies; c) studies utilizing animal models, cell cultures (in vitro or ex vivo), tissue-based cultures, and mRNA expression; d) and conference abstracts, case reports, editorials, comments or review articles, and articles that lack original data.

\section{Quality assessment of studies and data extraction}

The quality of observational studies was evaluated using the Newcastle-Ottawa quality assessment scale (NOS) described by Wells et al. [20]. The NOS tool includes nine items with scores ranging from 0 to 9. A higher score indicates a higher quality. Two investigators (JS and JR) independently reviewed the retrieved studies, and extracted the data to a table for review by a third investigator (CZ). Disagreements between investigators were resolved by discussion among all investigators. The first author's name, study sample size, year of publication, geographic region, study design, ethnicity, trimester in which the adipokine assays were performed, age, body mass index (BMI) and adipokine concentration in GDM patients (all means \pm standard deviation) were collected.

\section{Statistical analysis}

Statistical analysis was performed using STATA version 12.0 (Stata Corporation, College Station, TX, USA). The study effect sizes were described by SMDs and 95\% CIs. The results were reported as means and SD in most studies. A few studies reported results as medians with maxima and minima or medians and interquartile range. For those studies, the raw data were transformed to estimate the mean and SD. The method and its accuracy have been described elsewhere [21, 22]. Cochrane's $Q$ (chi square test) and the $I^{2}$ metric $\left(I^{2}=[(Q-\mathrm{df}) / Q) \times\right.$ $100 \%$ ] were used to assess heterogeneity. $I^{2}$ values of 25 , 50 , and $75 \%$ indicated low, medium, and high heterogeneity, respectively. A random-effect model (the DerSimonian and Laird method) was used in cases of heterogeneity, otherwise a fixed-effect model (the Mantel-Haenszel method) was used. Subgroup analysis and meta-regression analysis were used to determine the source of heterogeneity. Funnel plots, Begg's test and Egger's test were used to detect publication bias, with sensitivity analysis to identify the outliers if there was high heterogeneity. $P$-values $<0.05$ were considered statistically significant. 


\section{Results}

Literature research and characteristics of the included studies

Of the 494 articles that were retrieved, 20 were included in the meta-analysis; four were apelin studies $[4,6,7$, $11]$, nine were chemerin studies [5, 8, 15, 16, 23-27], five were omentin studies [28-32], one study involved both apelin and omentin [12], and one study involved both chemerin and omentin [33]. The 20 studies enrolled 2981 participants, of which 1493 were GDM patients and 1488 were healthy pregnant women (controls) [4-8, 11, 12, 15, 16, 23-33] (Fig. 1). The five apelin studies included 336 GDM patients and 237 controls. The 10 chemerin studies included 772 GDM patients and 857 controls. The five omentin studies included 385 GDM patients and 394 controls.

The 20 articles were published from 2010 to 2019. GDM was diagnosed based on the ADA criteria in five studies, the American College of Obstetricians and Gynecologists (ACOG) criteria in three studies, the Carpenter and Couston $(C \& C)$ criteria in two studies, the World Health Organization (WHO) criteria in three studies, the International Association of Diabetes and Pregnancy Study Group (IADPSG) criteria in four studies, the Australasian Diabetes in Pregnancy Society (ADIPS) criteria in two studies, and National Diabetes Date Group (NDDG) criteria in one study. Apelin, chemerin, and omentin were measured by enzyme linked

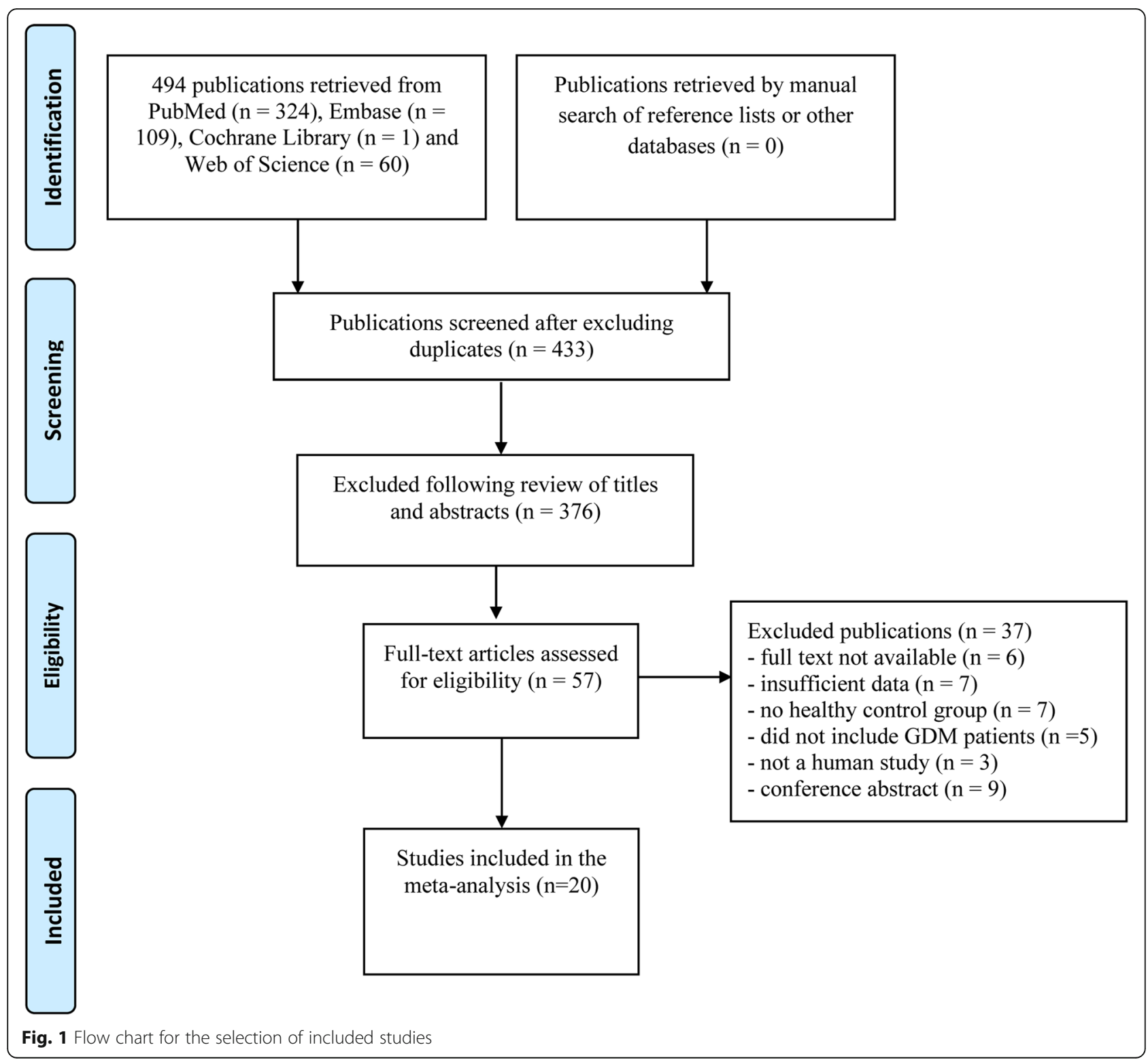


immunosorbent assay (ELISA) in 18 studies and by radioimmunoassay in two studies. The NOS scores ranged from 6 to 8 . The characteristics of the 20 included studies are shown in Table 1.

\section{Overall effects}

The results showed that there was no significant difference between GDM patients and normal controls in circulating apelin $(\mathrm{SMD}=0.43,95 \% \mathrm{CI}-0.40$ to $1.26, P=$ 0.31 ) and chemerin (SMD $=0.77,95 \% \mathrm{CI}-0.07$ to 1.61 , $P=0.07)$ levels. Circulating omentin was significantly lower in women with GDM than in healthy controls $(\mathrm{SMD}=-0.72,95 \% \mathrm{CI}-1.26$ to $-0.19, P=0.007)$.

\section{Heterogeneity}

Significant heterogeneity was detected in the apelin $\left(I^{2}=\right.$ 94.5\%, $P<0.001)$, chemerin $\left(I^{2}=98.0 \%, P<0.001\right)$, and omentin $\left(I^{2}=90.8 \%, P<0.001\right)$ studies. The randomeffect model was used for the meta-analysis (Fig. 2).

\section{Subgroup analysis}

The results of subgroup analyses of the study results stratified by ethnicity, age, BMI, study type, measurement type, diagnostic criteria, and trimester that the adipokine measurements were performed are shown in Table 2. Apelin levels were lower in women with GDM diagnosed by the ADA criteria than in controls, but the difference was significant with some but not all the commercially available ELISA kits. Asian and African women, patients younger than 30 years of age, those with BMIs $\geq 28 \mathrm{~kg} / \mathrm{m}^{2}$, and those diagnosed using the ACOG criteria in the second trimester had higher circulating chemerin levels than controls. Chemerin levels in GDM patients and controls did not differ in the other subgroups. Omentin was lower in GDM patients than in controls in Caucasian women, patients younger than 30 years of age, those with a BMI $<28 \mathrm{~kg} / \mathrm{m}^{2}$, or those diagnosed in the second trimester of pregnancy. No differences in the other subgroups were significant. Subgroup analysis suggested that differences in the ELISA kits used in the various studies may be the primary source of heterogeneity in the reported serum apelin levels.

\section{Meta-regression}

Meta-regression analysis was used to further identify sources of heterogeneity for chemerin and omentin (Table 3). Explanatory covariates included publication year, geographic region, sample size, BMI, and gestational age. However, none of those covariates changed the correlation between serum chemerin or omentin levels and GDM risk.

\section{Publication bias and sensitivity analysis}

Funnel plot symmetry indicated no significant publication bias among the studies (Fig. 3 and Table 4). The removal of any individual study in the sensitivity analysis did not change the overall statistical significance, indicating that that the results were statistically robust and reliable (Fig. 4).

\section{Discussion}

The meta-analysis of the association of circulating apelin, chemerin, and omentin with GDM included 20 studies, and found that apelin and chemerin levels in women with GDM did not differ significantly from those of controls with normal glucose tolerance. Omentin levels were lower in GDM patients than in women with normal pregnancies. Sensitivity analysis indicated that the pooled results were stable when the data of each individual study were deleted in succession. We did not find significant publication bias.

Apelin and its G protein-coupled receptor (APJ, apelin-angiotensin receptor-like 1) regulate glucose homeostasis in humans by increasing both glucose uptake and insulin sensitivity [34]. The increase of apelin levels in type 2 diabetes mellitus (T2DM) or obesity indicates an association between apelinemia and impaired glucose regulation [35]. Apelin stimulates various signaling pathways that decrease both cAMP and cAMPinduced AMPK activation. AMPK is a regulator of intracellular energy metabolism [36]. Apelin has been shown to decrease glucose transport in enterocytes by inhibiting sodium-dependent glucose transporter (SGLT)-1 [37]. In this study, apelin may have indirectly promoted an increase in plasma glucose by inhibiting glucose transport.

Apelin is present in human breast milk and may promote growth and regulate energy production in lactating infants [38]. In a recent review of clinical studies investigating the relationship between apelin and GDM, nearly all of the studies showed that lower apelin levels occurred in women with GDM compared to those without GDM. However, a few studies reported that the level of apelin-36 was increased in GDM [18].

Consistent with studies by Oncul et al. and Beata et al., we found that there were no significant differences in circulating apelin levels between GDM patients and controls with normal glucose tolerance [7]. In women diagnosed with GDM following the ADA criteria, circulating apelin was lower than that in controls, indicating that the diagnostic criteria may have affected the results obtained in previous studies. In addition, our subgroup analysis found significant heterogeneity in serum apelin levels in GDM that was associated with ELISA kits. Differences in the sensitivity and detection limits of the various ELISA kits resulted in heterogenous results. We 


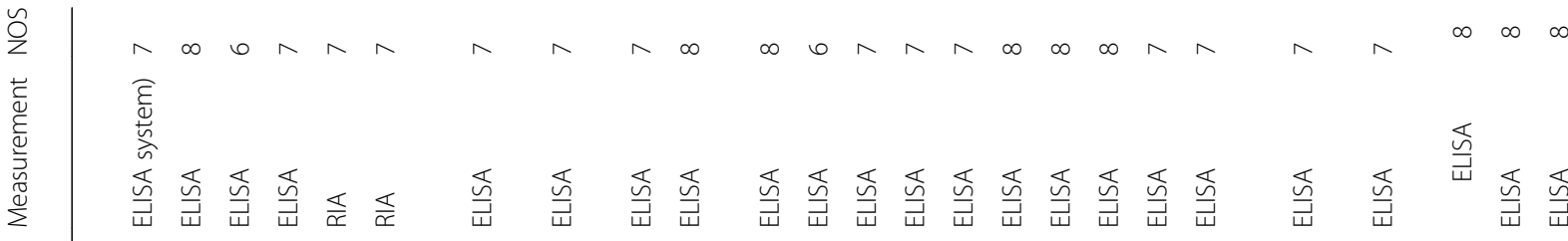

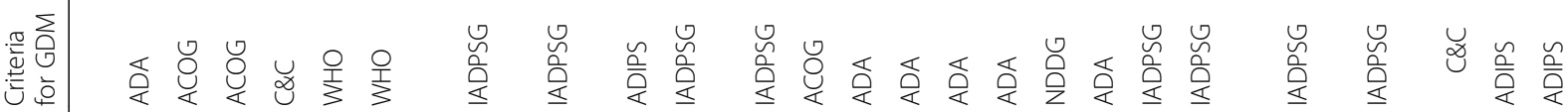

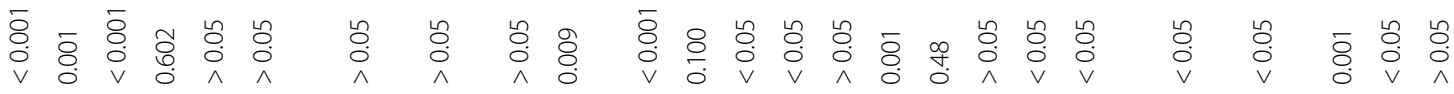

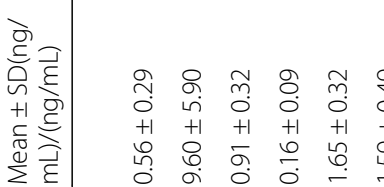

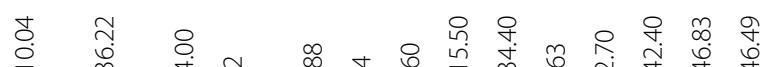

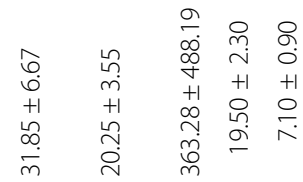

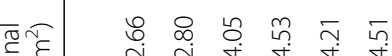

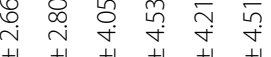

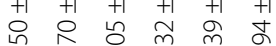

岂

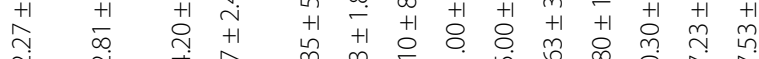

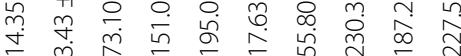

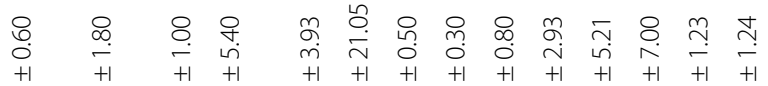

○.

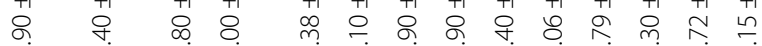

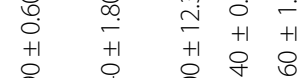

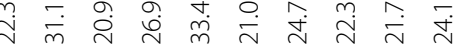

党

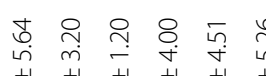

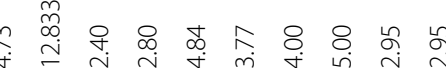

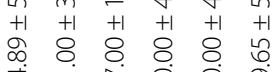

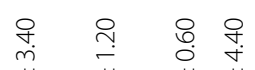

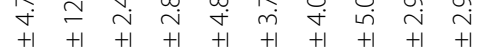

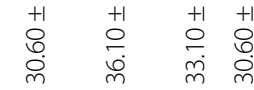

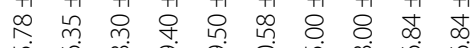

아 구

mi $\bar{n} \dot{m} \dot{m}$ iे

峲

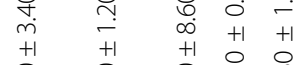

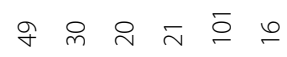

$\because \stackrel{\circ}{\circ}$

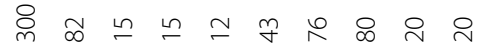

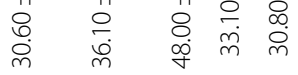

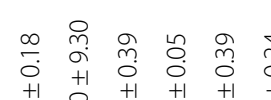

$\stackrel{8}{\circ}$

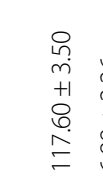

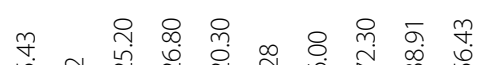

$\stackrel{\circ}{\sim} \hat{\sim}$

m.tr

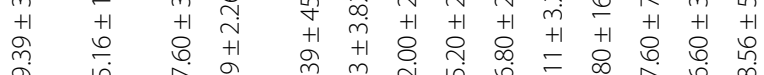

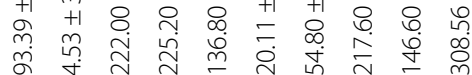

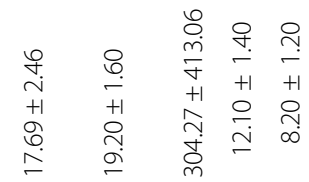

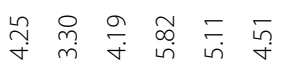

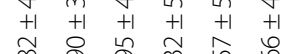

崩 山ें

œ

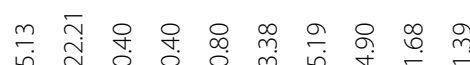

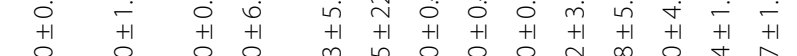

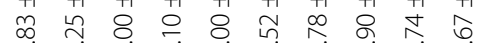

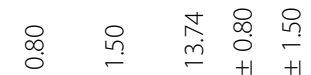

\begin{tabular}{llll}
$\circ$ & 8 & 0 & 0 \\
0 & 0 & \multicolumn{1}{c}{} & 0
\end{tabular}

ป্ं

+1
+
8
0

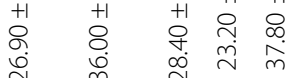

นึ ₹

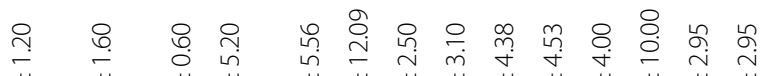

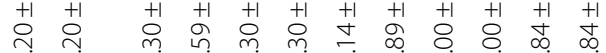

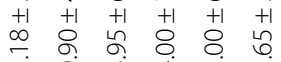

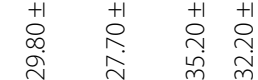

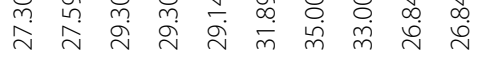

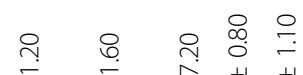

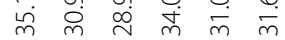

in ㅇำ

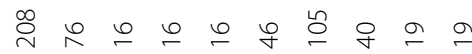

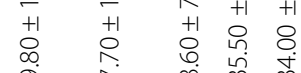

$z$

ఫ๐

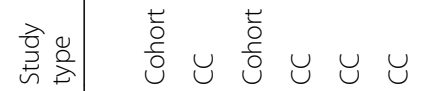

$\cup \quad \cup \quad \cup \cup$

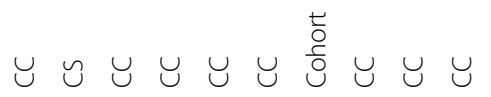

i $\bigcirc \stackrel{\infty}{\sim} \bar{\sim} \stackrel{\infty}{\bullet}$

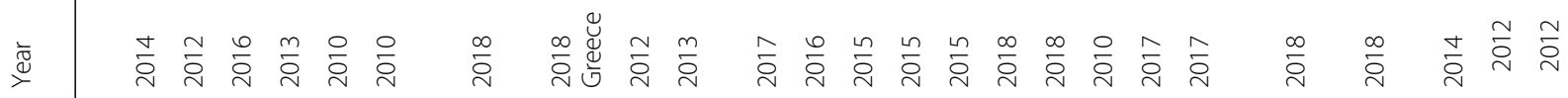




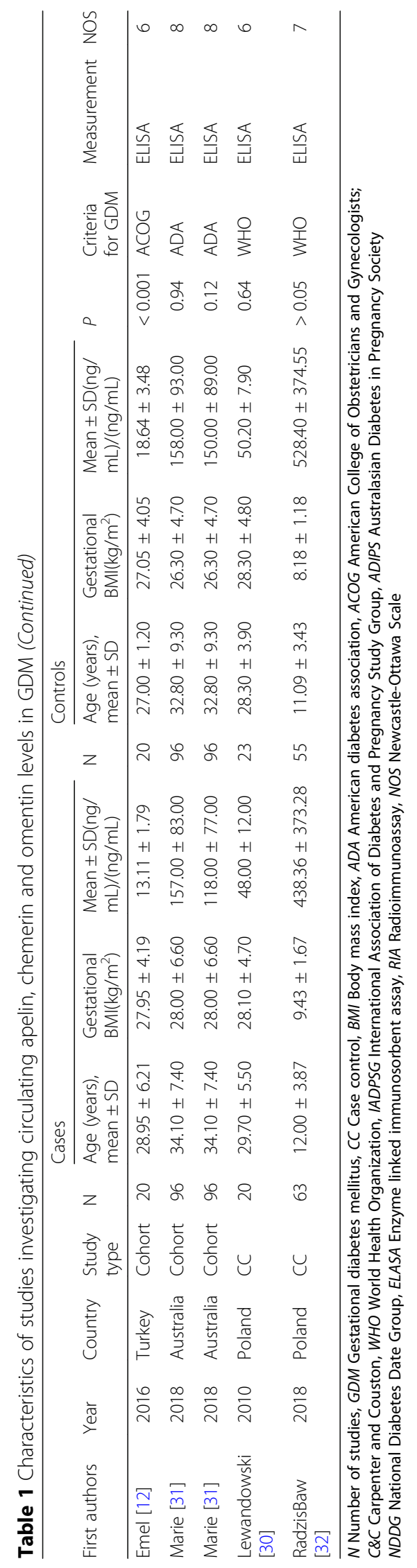




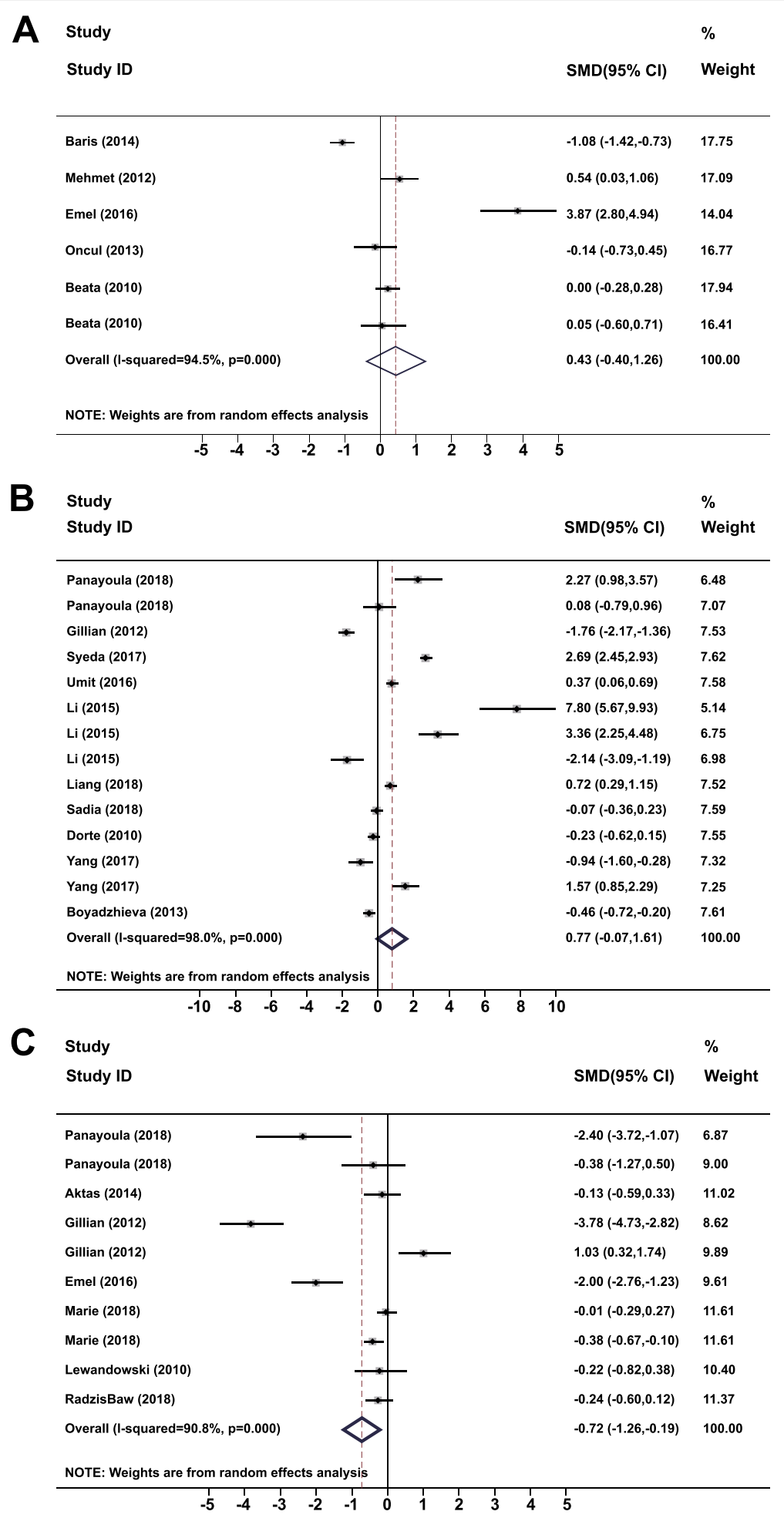

Fig. 2 Forest plots of heterogeneity in studies comparing serum (a) apelin (b) chemerin, and (c) omentin in GDM patients versus healthy controls 
Table 2 Stratified meta-analysis of circulating apelin, chemerin and omentin levels in GDM

\begin{tabular}{|c|c|c|c|c|c|c|c|}
\hline \multirow[t]{2}{*}{ Subgroups } & \multirow[t]{2}{*}{$\mathrm{N}$} & \multirow{2}{*}{$\begin{array}{l}\text { Test of association } \\
\text { SMD }(95 \% \mathrm{Cl})\end{array}$} & \multirow[b]{2}{*}{ z } & \multirow[b]{2}{*}{$P$} & \multicolumn{3}{|c|}{ Test of heterogeneity } \\
\hline & & & & & $\bar{Q}$ & $P^{2}(\%)$ & $P$ \\
\hline \multicolumn{8}{|l|}{ Apelin } \\
\hline \multicolumn{8}{|l|}{ Ethnicity } \\
\hline Caucasian & 2 & $0.01(-0.40$ to 1.26$)$ & 0.06 & 0.95 & 0.02 & 0.00 & 0.88 \\
\hline Asian & 4 & $0.72(-0.77$ to 2.22$)$ & 0.95 & 0.35 & 88.68 & 96.60 & $<0.05$ \\
\hline Combined & 6 & $0.43(-0.40$ to 1.26$)$ & 1.02 & 0.31 & 90.85 & 94.50 & $<0.05$ \\
\hline \multicolumn{8}{|c|}{ Age(mean,years) yyeyeyears) } \\
\hline$<30$ & 2 & $2.17(-1.09$ to 5.43$)$ & 1.31 & 0.19 & 30.23 & 88.20 & $<0.05$ \\
\hline$\geq 30$ & 4 & $-0.31(-0.93$ to 0.31$)$ & 0.99 & 0.32 & 25.41 & 96.70 & $<0.05$ \\
\hline Combined & 6 & $0.43(-0.40$ to 1.26$)$ & 1.02 & 0.31 & 90.85 & 94.50 & $<0.05$ \\
\hline \multicolumn{8}{|l|}{$\mathrm{BMI}\left(\right.$ mean, $\left.\mathrm{kg} / \mathrm{m}^{2}\right)$} \\
\hline$<28$ & 4 & $-0.14(-0.85$ to 0.57$)$ & 0.39 & 0.70 & 35.40 & 91.50 & $<0.05$ \\
\hline$\geq 28$ & 2 & $1.84(-2.09$ to 5.77$)$ & 0.92 & 0.36 & 41.58 & 97.60 & $<0.05$ \\
\hline Combined & 6 & $0.43(-0.40$ to 1.26$)$ & 1.02 & 0.31 & 90.85 & 94.50 & $<0.05$ \\
\hline \multicolumn{8}{|l|}{ Study type } \\
\hline Case-control & 4 & $0.10(-0.17$ to 0.36$)$ & 0.72 & 0.48 & 3.95 & 24.00 & 0.27 \\
\hline Cohort & 2 & $1.37(-3.48$ to 6.22$)$ & 0.55 & 0.58 & 74.68 & 98.70 & $<0.05$ \\
\hline Combined & 6 & $0.43(-0.40$ to 1.26$)$ & 1.02 & 0.31 & 90.85 & 94.50 & $<0.05$ \\
\hline \multicolumn{8}{|l|}{ Measurement type } \\
\hline ELISA & 4 & $0.72(-0.77$ to 2.22$)$ & 0.95 & 0.35 & 88.68 & 96.60 & $<0.05$ \\
\hline RIA & 2 & $0.01(-0.25$ to 0.26$)$ & 0.06 & 0.95 & 0.02 & 0.00 & 0.88 \\
\hline Combined & 6 & $0.43(-0.40$ to 1.26$)$ & 1.02 & 0.31 & 90.85 & 94.50 & $<0.05$ \\
\hline \multicolumn{8}{|l|}{ ELISA kits } \\
\hline Phoenix Pharmaceuticals & 1 & $-1.08(-1.42$ to -0.73$)$ & 6.16 & $<0.01$ & 0.02 & 0.00 & 0.23 \\
\hline Eastbiopharm & 1 & $0.54(0.03$ to 1.06$)$ & 2.06 & 0.04 & 25.41 & 25.00 & 0.87 \\
\hline Bio-Tek Instruments & 1 & 3.87 (2.80 to 4.94$)$ & 7.10 & $<0.01$ & 53.60 & 36.00 & 0.45 \\
\hline RayBiotech & 1 & $-0.14(-0.72$ to 0.45$)$ & 0.47 & 0.64 & 37.60 & 28.00 & 0.34 \\
\hline Combined & 4 & $0.72(-0.77$ to 2.22$)$ & 0.95 & 0.34 & 88.68 & 96.60 & $<0.05$ \\
\hline \multicolumn{8}{|l|}{ Diagnostic criteria } \\
\hline ADA & 1 & $-1.07(-1.42$ to -0.73$)$ & 6.16 & $<0.05$ & 0.00 & NA & NA \\
\hline ACOG & 2 & $2.17(-1.09$ to 5.43$)$ & 1.31 & 0.19 & 30.23 & 96.70 & $<0.05$ \\
\hline$C \& C$ & 1 & $-0.14(-0.73$ to 0.45$)$ & 0.47 & 0.64 & 0.00 & NA & NA \\
\hline WHO & 2 & $0.01(-0.25$ to 0.26$)$ & 0.06 & 0.95 & 0.02 & 0.00 & 0.88 \\
\hline Combined & 6 & $0.43(-0.40$ to 1.26$)$ & 1.02 & 0.31 & 90.85 & 94.50 & $<0.05$ \\
\hline \multicolumn{8}{|l|}{ Measurement trimester } \\
\hline Second & 1 & $0.00(-0.28$ to 0.28$)$ & 0.00 & 1.00 & 89.44 & 95.50 & $<0.05$ \\
\hline Third & 5 & $0.57(-0.62$ to 1.76$)$ & 0.94 & 0.35 & 0.00 & NA & NA \\
\hline Combined & 6 & $0.43(-0.40$ to 1.26$)$ & 1.02 & 0.31 & 90.85 & 94.50 & $<0.05$ \\
\hline \multicolumn{8}{|l|}{ Chemerin } \\
\hline \multicolumn{8}{|l|}{ Ethnicity } \\
\hline Caucasian & 5 & $-0.00(-0.44$ to 0.43$)$ & 0.01 & 0.99 & 19.03 & 79.00 & $<0.05$ \\
\hline Asian & 7 & 1.21 (0.09 to 2.35$)$ & 2.11 & 0.04 & 128.50 & 95.30 & $<0.05$ \\
\hline Australoid & 1 & $-1.76(-2.17$ to 1.36$)$ & 8.53 & 0.36 & 0.00 & NA & NA \\
\hline African & 1 & 2.69 (2.45 to 2.93$)$ & 21.74 & $<0.05$ & 0.00 & NA & NA \\
\hline
\end{tabular}


Table 2 Stratified meta-analysis of circulating apelin, chemerin and omentin levels in GDM (Continued)

\begin{tabular}{|c|c|c|c|c|c|c|c|}
\hline \multirow[t]{2}{*}{ Subgroups } & \multirow[t]{2}{*}{$N$} & \multirow{2}{*}{$\begin{array}{l}\text { Test of association } \\
\text { SMD }(95 \% \mathrm{Cl})\end{array}$} & \multirow[b]{2}{*}{ z } & \multirow[b]{2}{*}{$P$} & \multicolumn{3}{|c|}{ Test of heterogeneity } \\
\hline & & & & & $\bar{Q}$ & $P^{2}(\%)$ & $P$ \\
\hline Combined & 14 & $0.77(-0.07$ to 1.61$)$ & 1.80 & 0.07 & 651.01 & 98.00 & $<0.05$ \\
\hline \multicolumn{8}{|l|}{ Age(mean,years) } \\
\hline$<30$ & 9 & 1.51 (0.28 to 2.73$)$ & 2.41 & 0.02 & 306.13 & 97.40 & $<0.05$ \\
\hline$\geq 30$ & 5 & $-0.36(-1.03$ to 0.30$)$ & 1.07 & 0.28 & 75.12 & 94.70 & $<0.05$ \\
\hline Combined & 14 & $0.77(-0.07$ to 1.61$)$ & 1.80 & 0.07 & 651.01 & 98.00 & $<0.05$ \\
\hline \multicolumn{8}{|c|}{$\mathrm{BMI}\left(\right.$ mean, $\left.\mathrm{kg} / \mathrm{m}^{2}\right)$} \\
\hline$<28$ & 5 & $-0.75(-1.62$ to 0.11$)$ & 1.71 & 0.09 & 80.05 & 95.00 & $<0.05$ \\
\hline$\geq 28$ & 9 & 1.69 (0.58 to 2.79$)$ & 2.99 & $<0.05$ & 369.82 & 97.80 & $<0.05$ \\
\hline Combined & 14 & $0.77(-0.07$ to 1.61$)$ & 1.80 & 0.07 & 651.01 & 98.00 & $<0.05$ \\
\hline \multicolumn{8}{|l|}{ Study type } \\
\hline Case-control & 13 & $0.86(-0.08$ to 1.80$)$ & 1.79 & 0.07 & 636.04 & 98.10 & $<0.05$ \\
\hline Cohort & 1 & $-0.07(-0.36$ to 0.23$)$ & 0.45 & 0.65 & 0.00 & NA & NA \\
\hline Combined & 14 & $0.77(-0.07$ to 1.61$)$ & 1.80 & 0.07 & 651.01 & 98.00 & $<0.05$ \\
\hline \multicolumn{8}{|l|}{ ELISA kits } \\
\hline Millipore & 3 & $0.60(-0.50$ to 1.71$)$ & 1.07 & 0.29 & 11.90 & 83.20 & 0.29 \\
\hline R\&D systems & 4 & $-0.11(-1.64$ to 1.41$)$ & 0.15 & 0.88 & 100.69 & 97.00 & 0.88 \\
\hline Sbjbio & 3 & $2.94(-2.25$ to 0.12$)$ & 1.11 & 0.27 & 98.62 & 98.00 & 0.27 \\
\hline Biovendor & 2 & $0.08(-0.52$ to 0.67$)$ & 0.26 & 0.80 & 5.78 & 82.70 & 0.80 \\
\hline other kits & 2 & $1.11(-1.97$ to 4.20$)$ & 0.71 & 0.48 & 302.71 & 99.70 & 0.48 \\
\hline Combined & 14 & $0.77(-0.07$ to 1.61$)$ & 1.80 & 0.07 & 651.01 & 98.00 & 0.07 \\
\hline \multicolumn{8}{|c|}{ Diagnostic criteria } \\
\hline IADPSG & 6 & $0.86(-0.72$ to 2.43$)$ & 1.07 & 0.29 & 350.75 & 98.60 & $<0.05$ \\
\hline ADIPS & 1 & $1.26(-2.17$ to 1.36$)$ & 8.53 & $>0.05$ & 0.00 & NA & NA \\
\hline ACOG & 1 & $0.37(0.06$ to 0.69$)$ & 2.31 & 0.02 & 0.00 & NA & NA \\
\hline ADA & 4 & $2.27(-0.46$ to 4.99$)$ & 1.63 & 0.10 & 98.95 & 97.00 & $<0.05$ \\
\hline NDDG & 2 & $-0.13(-0.36$ to 0.10$)$ & 1.10 & 0.27 & 0.46 & 0.00 & 0.49 \\
\hline Combined & 14 & $0.77(-0.07$ to 1.61$)$ & 1.80 & 0.07 & 651.01 & 98.00 & $<0.05$ \\
\hline \multicolumn{8}{|c|}{ Measurement trimester } \\
\hline Second & 8 & $1.09(0.17$ to 2.34$)$ & 1.70 & 0.04 & 484.85 & 98.60 & $<0.05$ \\
\hline Third & 6 & $0.40(-0.61$ to 1.41$)$ & 0.77 & 0.44 & 112.32 & 95.50 & $<0.05$ \\
\hline Combined & 14 & $0.77(-0.07$ to 1.61$)$ & 1.80 & 0.07 & 651.01 & 98.00 & $<0.05$ \\
\hline \multicolumn{8}{|l|}{ Omentin } \\
\hline \multicolumn{8}{|l|}{ Ethnicity } \\
\hline Caucasian & 6 & $-0.33(-0.65$ to -0.02$)$ & 2.06 & 0.04 & 13.78 & 63.70 & 0.02 \\
\hline Asian & 2 & $-1.04(-2.87$ to 0.79$)$ & 1.11 & 0.27 & 16.82 & 94.10 & $<0.05$ \\
\hline Australoid & 2 & -1.36 (-6.07 to 3.35$)$ & 0.57 & 0.57 & 62.46 & 98.40 & $<0.05$ \\
\hline Combined & 10 & $-0.73(-1.26$ to -0.20$)$ & 2.68 & $<0.05$ & 97.49 & 90.80 & $<0.05$ \\
\hline \multicolumn{8}{|l|}{ Age(mean,years) } \\
\hline$<30$ & 6 & $-0.75(-1.36$ to -0.15$)$ & 2.44 & 0.02 & 27.75 & 82.00 & $<0.05$ \\
\hline$\geq 30$ & 4 & $-0.69(-1.73$ to 0.34$)$ & 1.31 & 0.19 & 68.06 & 95.60 & $<0.05$ \\
\hline Combined & 10 & $-0.73(-1.26$ to -0.20$)$ & 2.68 & $<0.05$ & 97.49 & 90.80 & $<0.05$ \\
\hline \multicolumn{8}{|c|}{ BMIImean,kg/m²) } \\
\hline$<28$ & 4 & $-2.06(-3.76$ to -0.37$)$ & 2.38 & 0.02 & 59.74 & 95.00 & $<0.05$ \\
\hline
\end{tabular}


Table 2 Stratified meta-analysis of circulating apelin, chemerin and omentin levels in GDM (Continued)

\begin{tabular}{|c|c|c|c|c|c|c|c|}
\hline \multirow[t]{2}{*}{ Subgroups } & \multirow[t]{2}{*}{ N } & \multirow{2}{*}{$\begin{array}{l}\text { Test of association } \\
\text { SMD }(95 \% \mathrm{Cl})\end{array}$} & \multirow[b]{2}{*}{ z } & \multirow[b]{2}{*}{$P$} & \multicolumn{3}{|c|}{ Test of heterogeneity } \\
\hline & & & & & $\bar{Q}$ & $P^{2}(\%)$ & $P$ \\
\hline$\geq 28$ & 6 & $-0.06(-0.38$ to 0.27$)$ & 0.35 & 0.73 & 14.48 & 65.50 & 0.01 \\
\hline Combined & 10 & $-0.73(-1.26$ to -0.20$)$ & 2.68 & $<0.05$ & 97.49 & 90.80 & $<0.05$ \\
\hline \multicolumn{8}{|l|}{ Study type } \\
\hline Case-control & 4 & $-0.57(-1.20$ to 0.05$)$ & 1.79 & 0.07 & 9.72 & 69.10 & 0.02 \\
\hline Cohort & 6 & $-0.79(-1.58$ to 0.10$)$ & 1.96 & 0.05 & 87.74 & 94.30 & $<0.05$ \\
\hline Combined & 10 & $-0.73(-1.26$ to 0.20$)$ & 2.68 & $>0.05$ & 97.49 & 90.80 & $<0.05$ \\
\hline \multicolumn{8}{|c|}{ Diagnostic criteria } \\
\hline IADPSG & 2 & $-1.33(-3.30$ to 0.65$)$ & 1.32 & 0.19 & 6.15 & 83.80 & 0.01 \\
\hline$C \& C$ & 2 & -1.04 ( -2.87 to 0.79$)$ & 1.11 & 0.27 & 16.82 & 94.10 & $<0.05$ \\
\hline ADIPS & 2 & $-1.36(-6.07$ to 3.35$)$ & 0.57 & 0.57 & 62.46 & 98.40 & $<0.05$ \\
\hline ADA & 2 & $-0.20(-0.56$ to 0.17$)$ & 1.06 & 0.29 & 3.31 & 69.80 & 0.07 \\
\hline $\mathrm{WHO}$ & 2 & $-0.24(-0.55$ to 0.08$)$ & 1.48 & 0.14 & 0.00 & 0.00 & 0.95 \\
\hline Combined & 10 & $-0.73(-1.26$ to -0.20$)$ & 2.68 & $<0.05$ & 97.49 & 90.80 & $<0.05$ \\
\hline \multicolumn{8}{|l|}{ ELISA kits } \\
\hline Millipore & 2 & $-1.33(-3.29$ to 0.65$)$ & 1.32 & 0.18 & 6.15 & 83.80 & 0.01 \\
\hline Cusabio & 2 & $-1.36(-6.07$ to 3.35$)$ & 0.57 & 0.57 & 62.46 & 98.40 & $<0.05$ \\
\hline Life Science & 2 & $-0.19(-0.56$ to 0.17$)$ & 1.06 & 0.29 & 3.31 & 69.80 & 0.06 \\
\hline Bio Vendor & 2 & $-1.09(-2.81$ to 0.64$)$ & 1.24 & 0.21 & 16.53 & 94.00 & $<0.05$ \\
\hline other kits & 2 & $-0.16(-0.53$ to 0.20$)$ & 0.88 & 0.38 & 0.05 & 0.00 & 0.82 \\
\hline Combined & 10 & $-0.73(-1.26$ to -0.19$)$ & 2.68 & $<0.05$ & 97.49 & 90.80 & $<0.05$ \\
\hline \multicolumn{8}{|c|}{ Measurement trimester } \\
\hline Second & 4 & $-0.12(-0.31$ to -0.08$)$ & 1.19 & 0.04 & 84.73 & 94.10 & $<0.05$ \\
\hline Third & 6 & $-1.27(-2.45$ to 0.08$)$ & 2.10 & 0.23 & 1.10 & 0.00 & 0.78 \\
\hline Combined & 10 & $-0.73(-1.26$ to -0.20$)$ & 2.68 & $<0.05$ & 97.49 & 90.80 & $<0.05$ \\
\hline
\end{tabular}

$N$ Number of studies, SMD Standardized mean difference, BMI Body mass index, ELASA Enzyme linked immunosorbent assay, RIA Radioimmunoassay, NA Not available

Table 3 Univariate meta-regression analysis of heterogeneity caused by patient variables across studies

\begin{tabular}{|c|c|c|c|c|c|}
\hline Variables & Coefficient & Standard error & $95 \% \mathrm{Cl}$ & $t$ & $P$ \\
\hline \multicolumn{6}{|l|}{ Chemerin } \\
\hline Publication year & 0.19 & 0.27 & {$[-0.40,0.79]$} & 0.71 & 0.50 \\
\hline Geographic region & 0.37 & 3.18 & {$[-7.40,8.15]$} & 0.12 & 0.91 \\
\hline Sample size & 0.98 & 0.96 & {$[-1.11,3.07]$} & 1.02 & 0.33 \\
\hline $\mathrm{BMl}$ & 6.39 & 3.80 & {$[-1.89,14.68]$} & 1.68 & 0.12 \\
\hline Gestational age & 8.26 & 6.89 & {$[-6.77,23.29]$} & 1.20 & 0.25 \\
\hline \multicolumn{6}{|l|}{ Omentin } \\
\hline Publication year & -18.92 & 317.55 & {$[-751.20,713.36]$} & -0.06 & 0.95 \\
\hline Geographic region & -1.05 & 1.22 & {$[-4.19,2.09]$} & -0.86 & 0.43 \\
\hline Sample size & -1.33 & 0.70 & {$[-2.93,0.28]$} & -1.91 & 0.09 \\
\hline $\mathrm{BMl}$ & -2.10 & 1.74 & {$[-6.11,1.90]$} & -1.21 & 0.26 \\
\hline Gestational age & 0.17 & 2.13 & {$[-4.74,5.09]$} & 0.08 & 0.94 \\
\hline
\end{tabular}



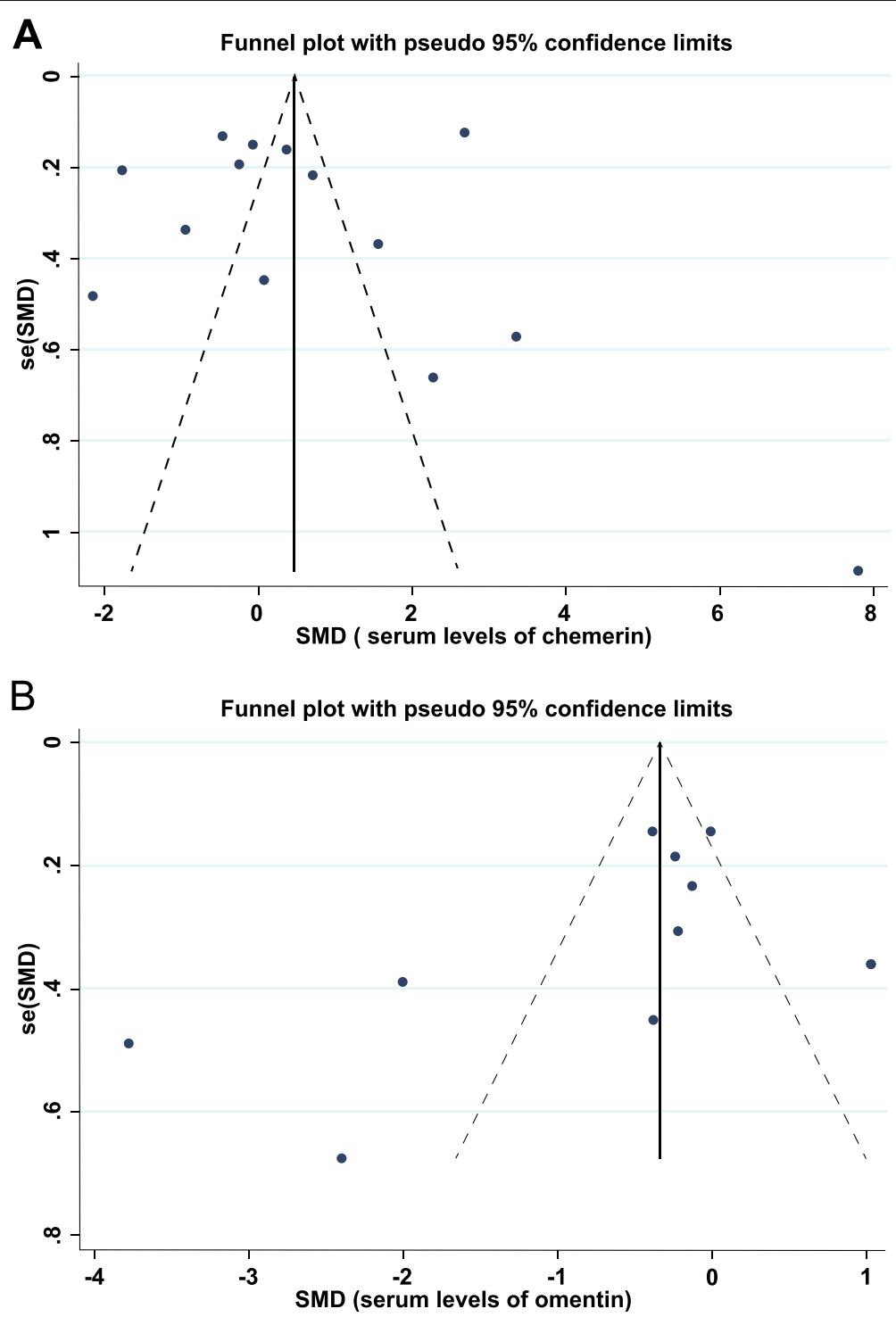

Fig. 3 Funnel plots for estimating publication bias in studies of (a) chemerin and (b) omentin in women with GDM

found that the year of publication, geographic region, study sample size, BMI, and gestational age were not sources of heterogeneity across the included studies.

The binding of chemerin to its receptors can promote the recruitment of macrophages and dendritic cells, and neutrophil activation to produce inflammation [39], and chronic low-grade inflammation has been associated with increased IR [40]. A reduction in chemerin levels may be associated with the development of GDM through decreased insulin sensitivity and attenuated anti-inflammatory capacity [25].

The finding in this meta-analysis that circulating chemerin levels were similar in women with GDM and in normal pregnant women is consistent with previous

Table 4 Heterogeneity and publication bias in studies of serum apelin, chemerin and omentin in women with GDM

\begin{tabular}{|c|c|c|c|c|c|c|c|c|}
\hline \multirow[b]{2}{*}{ Adipokine in GDM } & \multirow[b]{2}{*}{$\mathrm{N}$} & \multirow[b]{2}{*}{$\operatorname{SMD}(95 \% \mathrm{Cl})$} & \multicolumn{2}{|c|}{ Heterogeneity test } & \multirow[b]{2}{*}{$t$} & \multirow{2}{*}{$\begin{array}{l}\text { Egger's test } \\
P\end{array}$} & \multirow[b]{2}{*}{$z$} & \multirow{2}{*}{$\begin{array}{l}\text { Begg's test } \\
P\end{array}$} \\
\hline & & & $P^{2}$ & $P$ & & & & \\
\hline Apelin in GDM & 6 & $0.43(-0.40$ to 1.26$)$ & 94.5 & $<0.001$ & 1.66 & 0.17 & 0.94 & 0.35 \\
\hline Chemerin in GDM & 14 & $0.77(-0.07$ to 1.61$)$ & 98.0 & $<0.001$ & 0.03 & 0.98 & 1.26 & 0.21 \\
\hline Omentin in GDM & 10 & $-0.72(-1.26$ to -0.19$)$ & 90.8 & $<0.001$ & -1.73 & 0.12 & -1.70 & 0.09 \\
\hline
\end{tabular}

$N$ Number of studies, SMD Standardized mean difference 


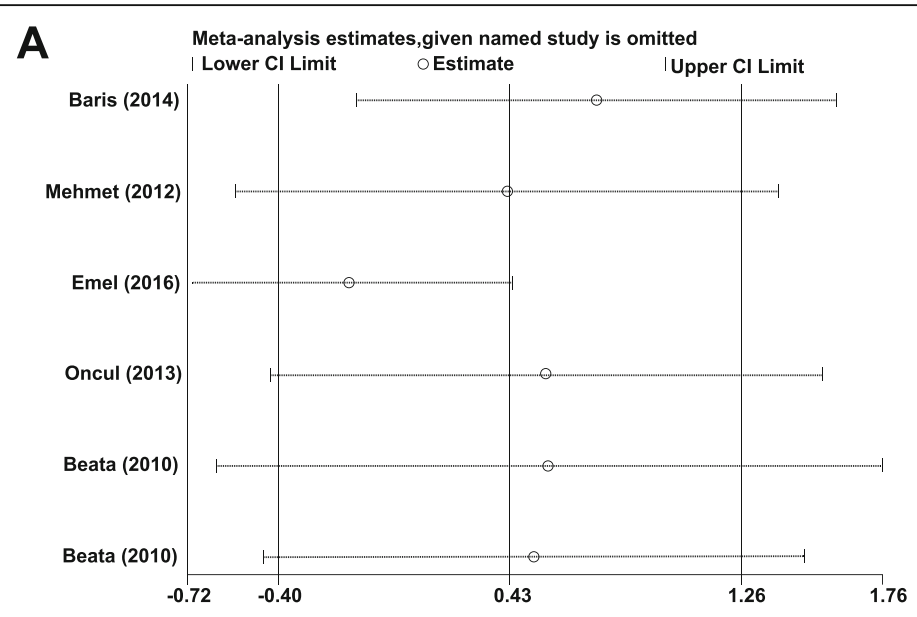

B
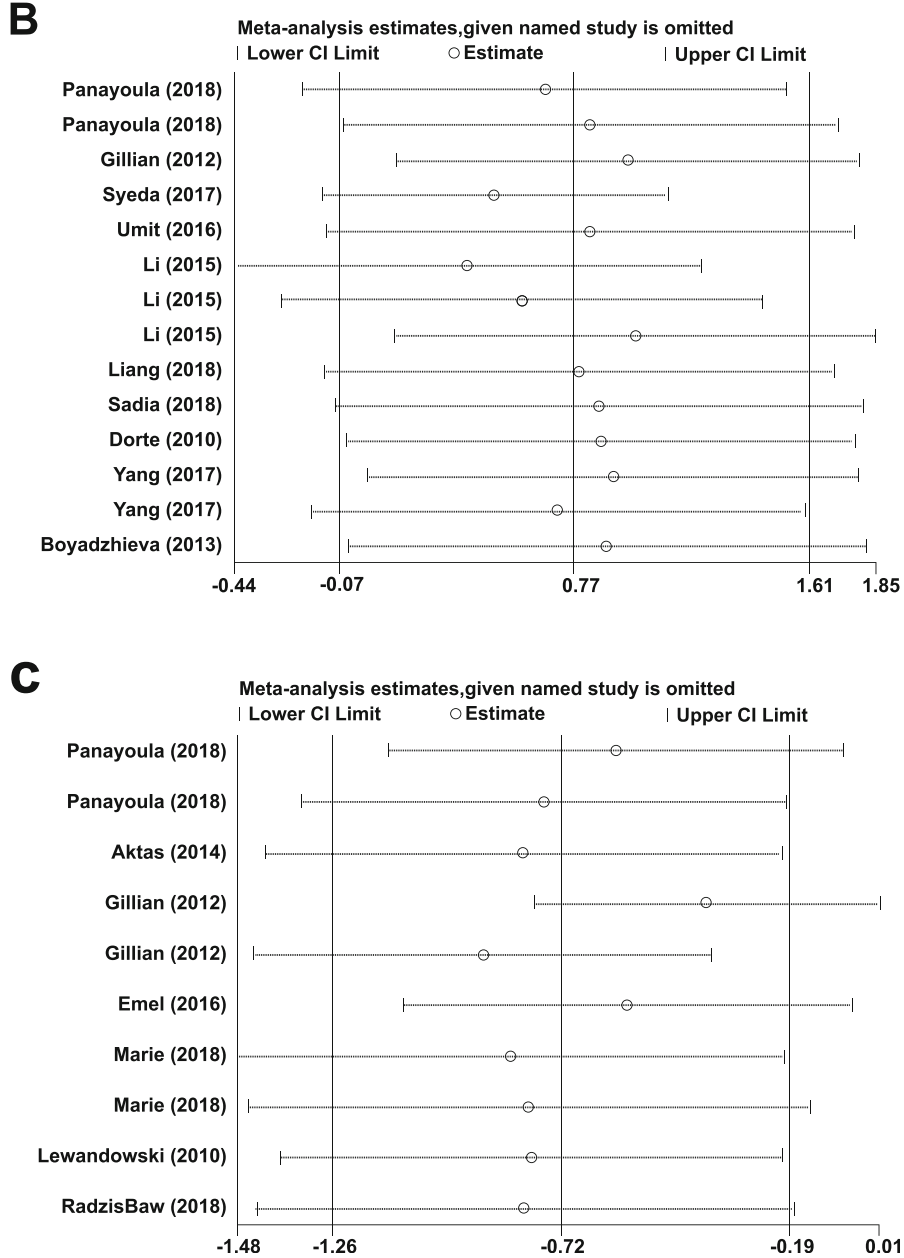

Fig. 4 Sensitivity analysis plots of differences in serum (a) apelin (b) chemerin, and (c) omentin levels in GDM patients and healthy controls

studies [8, 23]. Unlike Zhou et al., this meta-analysis took ethnicity, diagnostic criteria, and sample size into account [41]. Sub-analysis revealed that Asian and African women, patients younger than 30 years of age, those with BMIs $\geq 28 \mathrm{~kg} / \mathrm{m}^{2}$, or those diagnosed using the
ACOG criteria in the second trimester had higher circulating chemerin levels than controls. Firstly, women with GDM and high BMIs had significantly higher chemerin concentrations than normal-weight women with GDM, consistent with a positive correlation of chemerin level 
and BMI. Obesity may have caused an increase in chemerin in pregnant women before delivery [15]. The results are consistent with those of Van Poppel et al., who found that circulating chemerin in pregnancy was influenced by maternal obesity status rather than GDM [42].

Secondly, the subgroup analysis also found that GDM patients younger than 30 years of age had a mean chemerin concentration 1.51 times that of normal controls, which indicates a negative correlation of age and chemerin concentration in GDM. Thirdly, chemerin levels were higher in GDM diagnosed during the second trimester than in controls. This result differs from that of Yang et al. who reported higher circulating chemerin in the third trimester than in early pregnancy [15]. A recent study found that both chemerin released by adipocytes and albumen decreased in late pregnancy to accommodate the increased nutrition needs of the fetus, which might partially explain why GDM patients had lower chemerin levels in the third trimester [43].

Omentin may influence plasma glucose concentration by promoting insulin-stimulated glucose uptake in human subcutaneous and visceral adipocytes [15]. Pan et al. found that circulating omentin after fasting and $2 \mathrm{~h}$ post-glucose load were significantly decreased in patients with impaired glucose tolerance, and in those with newly diagnosed, untreated diabetes compared to healthy controls [44]. Similarly, El-Mesallamy et al. reported decreased circulating omentin levels in T2DM patients after adjusting for age or BMI [45]. The association of omentin and diabetes is not limited to patients with T2DM. Polkowska et al. revealed that circulating omentin concentration was lower in children with T1DM than in control children [46].

However, the data on omentin concentrations in GDM are limited. Our meta-analysis found that circulating omentin was lower in GDM patients than in controls. Interestingly, omentin is thought to be primarily expressed in visceral adipose tissue; however, in this analysis, omentin level was shown to be negatively associated with the amount of visceral adipose tissue. Visceral obesity has also been found to be associated with omentin secretion in adipose tissue. During the accretion of visceral fat, the release of free fatty acids and inflammatory cytokines into the portal vein increases, which leads to increased oxidative stress and IR. A decrease in omentin concentration, released from visceral adipose tissue might generate IR that causes GDM [32].

The subgroup analysis found that Caucasian, but not Asian or Australoid GDM patients had lower circulating omentin levels than controls. Circulating omentin levels were significantly higher in GDM patients younger than 30 years of age than in controls, and were significantly different in GDM patients with BMIs $<28 \mathrm{~kg} / \mathrm{m}^{2}$ than in controls. Both age and BMI might influence circulating omentin concentrations in GDM patients. In homologous groups, omentin levels were higher in the second trimester (approximately 28 weeks gestation) than in the third trimester. The difference might have been the result of omentin production in both visceral adipose tissue and the placenta, which is the major source of omentin during pregnancy [32]. Thus, there will be increased omentin levels in early pregnancy, and omentin clearance during the later stages of pregnancy. Second, omentin may be increased in the second trimester of pregnancy because of increased fat accretion or decreased secretion from maternal adipose tissue [47-49].

Several study limitations should be taken into consideration. We excluded grey literature, such as conference abstracts and experimental animal studies, which may have led to selection bias. Heterogeneity may have influenced the interpretation of the results. There was substantial heterogeneity across studies, although randomeffects models were applied to avoid statistical heterogeneity. Subgroup analysis of data stratified by seven potential sources also confirmed study heterogeneity (Table 2). However, sensitivity analysis and metaregression analysis may have failed to identify all sources of heterogeneity because of insufficient data. Heterogeneity might also reflect differences in clinical variables, such as drug use, smoking status, alcohol consumption, exercise, environmental factors, diet adjustment, or an ideal index for reflecting obesity such as waist-hip ratio or waist circumference that were not considered. Considering the limited number of studies included in this meta-analysis, the relationship between circulating omentin levels and GDM requires further investigation.

\section{Conclusions}

The results of this meta-analysis support omentin as a novel biomarker for the early diagnosis of GDM, which affects many pregnant women. Omentin deficiency may be involved in the pathogenesis of GDM. The decreased omentin levels in mothers with GDM, compared with healthy controls may result from impaired synthesis or release, but the mechanism for this requires further investigation. To date, the precise mechanisms by which omentin plays a role in glucose metabolism are not understood. In the future, we will carry out a large-scale prospective study to address the limitations.

\section{Supplementary information}

Supplementary information accompanies this paper at https://doi.org/10. 1186/s12944-020-01209-7.

Additional file 1. A full description of the search terms and strategy.

\section{Abbreviations}

ACOG: American College of Obstetricians and Gynecologists; ADA: American diabetes association; ADIPS: Australasian Diabetes in Pregnancy Society; 
APJ: Apelin-angiotensin receptor-like 1; BMI: Body mass index; C\&C: Carpenter and Couston; Cl: Confidence interval; ELISA: Enzyme linked immunosorbent assay; GDM: Gestational diabetes mellitus; IADPSG: International Association of Diabetes and Pregnancy Study Group: IR: Insulin resistance; NDDG: National Diabetes Date Group; NOS: NewcastleOttawa quality assessment scale; PRISMA: Preferred Reporting Items for Systematic Reviews and Meta-analysis; RARRES2: Retinoic acid receptor responder protein 2; SD: Standard deviation; SGLT-1: Sodium-dependent glucose transporter-1; SMD: Standardized mean difference; T1DM: Type 1 diabetes; T2DM: Type 2 diabetes; TIG2: Tazarotene-induced gene 2 protein; WHO: World Health Organization

\section{Acknowledgements}

Not applicable.

\section{Authors' contributions}

JS and JR performed the literature search, data extraction, quality assessment, and drafted the manuscript. CZ contributed to editing and revision of the manuscript. SY designed the study, interpreted the data and revised the manuscript. All authors read and approved the final manuscript.

\section{Funding}

This study was supported by grants from the National Natural Science Foundation of China (30771849, 30972530, 81273169,81573218 and 81773514).

\section{Availability of data and materials}

All data generated or analyzed during this study are included in this article.

\section{Ethics approval and consent to participate}

Not applicable.

\section{Consent for publication}

Not applicable.

\section{Competing interests}

The authors declare that they have no competing interests.

\section{Author details}

${ }^{1}$ Division of Life Science and Medicine, Department of Endocrinology, The First Affiliated Hospital of USTC (Anhui Provincial Hospital), University of Science and Technology of China, 17 Lujiang Road, Hefei 230001, China. ${ }^{2}$ Department of Rheumatology and Immunology, Arthritis Research Institute, The First Affiliated Hospital of Anhui Medical University, 218 Jixi Road, Hefei 230022, Anhui, China. ${ }^{3}$ Department of Endocrinology, Institute of Endocrinology and Metabolism, The First Affiliated Hospital of Anhui Medical University, 218 Jixi Road, Hefei 230022, Anhui, China. ${ }^{4}$ Department of Epidemiology and Biostatistics, School of Public Health, Anhui Medical University, 81Meishan Road, Hefei 230032, Anhui, China.

\section{Received: 16 November 2019 Accepted: 18 February 2020}

Published online: 22 February 2020

\section{References}

1. American Diabetes Association. Classification and diagnosis of diabetes: standards of medical Care in Diabetes-2019. Diabetes Care. 2019;42:S13-28.

2. Fasshauer $M$, Bluher $M$, Stumvoll $M$. Adipokines in gestational diabetes. Lancet Diabetes Endocrinol. 2014;2:488-99.

3. Jaffe A, Giveon S, Rubin C, Novikov I, Ziv A, Kalter-Leibovici O. Gestational diabetes risk in a multi-ethnic population. Acta Diabetol. 2019. https://doi. org/10.1007/s00592-019-01404-8 [Epub ahead of print].

4. Telejko B, Kuzmicki M, Wawrusiewicz-Kurylonek N, Szamatowicz J, Nikolajuk A, Zonenberg A, Zwierz-Gugala D, Jelski W, Laudanski P, Wilczynski J, Kretowski A, Gorska M. Plasma apelin levels and apelin/APJ mRNA expression in patients with gestational diabetes mellitus. Diabetes Res Clin Pract. 2010;87:176-83.

5. Boyadzhieva M, Atanasova I, Zacharieva S, Kedikova S. Adipocytokines during pregnancy and postpartum in women with gestational diabetes and healthy controls. J Endocrinol Investig. 2013;36:944-9.
6. Aslan M, Celik O, Celik N, Turkcuoglu I, Yilmaz E, Karaer A, Simsek Y, Celik E, Aydin S. Cord blood nesfatin-1 and apelin-36 levels in gestational diabetes mellitus. Endocrine. 2012;41:424-9.

7. Oncul M, Tuten A, Erman H, Gelisgen R, Benian A, Uzun H. Maternal and cord blood apelin, resistin and visfatin levels in gestational diabetes mellitus. Minerva Med. 2013;104:527-35.

8. Pfau D, Stepan H, Kratzsch J, Verlohren M, Verlohren HJ, Drynda K, Lossner U, Bluher M, Stumvoll M, Fasshauer M. Circulating levels of the adipokine chemerin in gestational diabetes mellitus. Horm Res Paediatr. 2010;74:56-61.

9. Mastrella G, Hou M, Li M, Stoecklein VM, Zdouc N, Volmar MNM, Miletic H, Reinhard S, Herold-Mende CC, Kleber S, Eisenhut K, Gargiulo G, Synowitz M, Vescovi AL, Harter PN, Penninger JM, Wagner E, Mittelbronn M, Bjerkvig R, Hambardzumyan D, Schuller U, Tonn JC, Radke J, Glass R, Kalin RE. Targeting APLN/APLNR improves Antiangiogenic efficiency and blunts Proinvasive side effects of VEGFANEGFR2 blockade in Glioblastoma. Cancer Res. 2019; 79:2298-313.

10. Elsehmawy A, El-Toukhy SE, Seliem NMA, Moustafa RS, Mohammed DS. Apelin and chemerin as promising adipokines in children with type 1 diabetes mellitus. Diabetes Metab Syndr Obes. 2019;12:383-9.

11. Akinci B, Celtik A, Tunali S, Genc S, Yuksel F, Secil M, Ozcan MA, Bayraktar F. Circulating apelin levels are associated with cardiometabolic risk factors in women with previous gestational diabetes. Arch Gynecol Obstet. 2014;289:78793.

12. Kiyak Caglayan E, Engin-Ustun Y, Sari N, Gocmen AY, Polat MF. The effects of prolonged fasting on the levels of adiponectin, leptin, apelin, and omentin in pregnant women. J Obstet Gynaecol. 2016;36:555-8.

13. Estienne A, Bongrani $A$, Reverchon $M$, Rame C, Ducluzeau PH, Froment $P$, Dupont J. Involvement of Novel Adipokines, Chemerin, Visfatin, Resistin and Apelin in Reproductive Functions in Normal and Pathological Conditions in Humans and Animal Models. Int J Mol Sci. 2019. p. 20. https://doi.org/10. 3390/ijms20184431.

14. Wittamer V, Franssen JD, Vulcano M, Mirjolet JF, Le Poul E, Migeotte I, Brezillon S, Tyldesley R, Blanpain C, Detheux M, Mantovani A, Sozzani S, Vassart G, Parmentier M, Communi D. Specific recruitment of antigenpresenting cells by chemerin, a novel processed ligand from human inflammatory fluids. J Exp Med. 2003;198:977-85.

15. Yang X, Quan X, Lan Y, Ye J, Wei Q, Yin X, Fan F, Xing H. Serum chemerin level during the first trimester of pregnancy and the risk of gestational diabetes mellitus. Gynecol Endocrinol. 2017;33:770-3.

16. Mehmood S, Ye C, Connelly PW, Hanley AJ, Zinman B, Retnakaran R. Rising plasminogen activator inhibitor-1 and hypoadiponectinemia characterize the cardiometabolic biomarker profile of women with recent gestational diabetes. Cardiovasc Diabetol. 2018;17:133.

17. Tok A, Ozer A, Kanat-Pektas M, Aral M, Sakalli H, Aydogdu S, Yutan-Kaya E, Sager $\mathrm{H}$. The role of omentin in early pregnancy losses. J Obstet Gynaecol. 2019:40:107-10. https://doi.org/10.1080/01443615.2019.1606179.

18. De Gennaro G, Palla G, Battini L, Simoncini T, Del Prato S, Bertolotto A, Bianchi $C$. The role of adipokines in the pathogenesis of gestational diabetes mellitus. Gynecol Endocrinol. 2019;35:737-51.

19. Tam WWS, Tang A, Woo B, Goh SYS. Perception of the preferred reporting items for systematic reviews and meta-analyses (PRISMA) statement of authors publishing reviews in nursing journals: a cross-sectional online survey. BMJ Open. 2019;9:e026271.

20. Wells GA, Shea BJ, O'Connell D, Peterson J, Welch V, Losos M, Tugwell P. The Newcastle-Ottawa scale (NOS) for assessing the quality of nonrandomized studies in meta-analysis. Appl Eng Agric. 2014;18:727-34.

21. Wan X, Wang W, Liu J, Tong T. Estimating the sample mean and standard deviation from the sample size, median, range and/or interquartile range. BMC Med Res Methodol. 2014;14:135.

22. Luo D, Wan X, Liu J, Tong T. Optimally estimating the sample mean from the sample size, median, mid-range, and/or mid-quartile range. Stat Methods Med Res. 2018;27:1785-805.

23. Barker G, Lim R, Rice GE, Lappas M. Increased chemerin concentrations in fetuses of obese mothers and correlation with maternal insulin sensitivity. J Matern Fetal Neonatal Med. 2012;25:2274-80.

24. Li XM, Ji H, Li CJ, Wang PH, Yu P, Yu DM. Chemerin expression in Chinese pregnant women with and without gestational diabetes mellitus. Ann Endocrinol (Paris). 2015;76:19-24.

25. Liang Z, Zhou M, Xu XK, Qu F, Chen D. Is Chemerin associated with gestational diabetes mellitus? An evidence-based clinical research from Chinese women. J Obstet Gynaecol. 2018;38:482-7. 
26. Fatima SS, Alam F, Chaudhry B, Khan TA. Elevated levels of chemerin, leptin, and interleukin-18 in gestational diabetes mellitus. J Matern Fetal Neonatal Med. 2017;30:1023-8.

27. Gorkem U, Kucukler FK, Togrul C, Gungor T. Are adipokines associated with gestational diabetes mellitus? J Turk Ger Gynecol Assoc. 2016;17:186-90.

28. Aktas G, Alcelik A, Ozlu T, Tosun M, Tekce BK, Savli H, Tekce H, Dikbas O. Association between omentin levels and insulin resistance in pregnancy. Exp Clin Endocrinol Diabetes. 2014;122:163-6.

29. Barker G, Lim R, Georgiou HM, Lappas M. Omentin-1 is decreased in maternal plasma, placenta and adipose tissue of women with pre-existing obesity. PLoS One. 2012;7:e42943.

30. Lewandowski K, Nadel I, Lewinski A, Bienkiewicz M, Tan B, Randeva HS, Cypryk K. Positive correlation between serum omentin and thrombospondin-1 in gestational diabetes despite lack of correlation with insulin resistance indices. Ginekol Pol. 2010;81:907-12.

31. Franz M, Polterauer M, Springer $S$, Kuessel L, Haslinger P, Worda C, Worda K. Maternal and neonatal omentin-1 levels in gestational diabetes. Arch Gynecol Obstet. 2018;297:885-9.

32. Mierzynski R, Dluski D, Nowakowski L, Poniedzialek-Czajkowska E, Leszczynska-Gorzelak B. Adiponectin and Omentin levels as predictive biomarkers of preterm birth in patients with gestational diabetes mellitus. Biomed Res Int. 2018;2018:7154216.

33. Tsiotra PC, Halvatsiotis P, Patsouras K, Maratou E, Salamalekis G, Raptis SA, Dimitriadis $G$, Boutati E. Circulating adipokines and mRNA expression in adipose tissue and the placenta in women with gestational diabetes mellitus. Peptides. 2018;101:157-66.

34. Waller JD, McNeill EH, Zhong F, Vervaecke LS, Goldfarb AH. Plasma Apelin unchanged with acute exercise insulin sensitization. J Sports Sci Med. 2019; 18:537-43.

35. Norvezh F, Razi Jalali M, Tabandeh MR, Hajikolaei MRH, Gooraninejad S. Serum Apelin-36 alteration in late pregnancy and early lactation of dairy cows and its association with negative energy balance markers. Res Vet Sci. 2019;125:285-9.

36. Tekin S, Erden Y, Sandal S, Etem Onalan E, Ozyalin F, Ozen H, Yilmaz B. Effects of apelin on reproductive functions: relationship with feeding behavior and energy metabolism. Arch Physiol Biochem. 2017:123:9-15.

37. Bertrand C, Valet P, Castan-Laurell I. Apelin and energy metabolism. Front Physiol. 2015;6:115

38. Aydin S. The presence of the peptides apelin, ghrelin and nesfatin-1 in the human breast milk, and the lowering of their levels in patients with gestational diabetes mellitus. Peptides. 2010;31:2236-40.

39. Dellepiane S, Medica D, Guarena C, Musso T, Quercia AD, Leonardi G, Marengo M, Migliori M, Panichi V, Biancone L, Pizzarelli F, Camussi G, Cantaluppi V. Citrate anion improves chronic dialysis efficacy, reduces systemic inflammation and prevents Chemerin-mediated microvascular injury. Sci Rep. 2019;9:10622.

40. Gong Z, Zhang X, Su K, Jiang R, Sun Z, Chen W, Forno E, Goetzman ES, Wang J, Dong HH, Dutta P, Muzumdar R. Deficiency in AIM2 induces inflammation and adipogenesis in white adipose tissue leading to obesity and insulin resistance. Diabetologia. 2019. https://doi.org/10.1007/s00125019-04983-x.

41. Zhou Z, Chen H, Ju H, Sun M. Circulating chemerin levels and gestational diabetes mellitus: a systematic review and meta-analysis. Lipids Health Dis. 2018;17:169.

42. van MNM P, Willibald Z, Daniela U, Eva-Christina S, Birgit H, Uwe L, Christian W, Gernot D. Cord blood chemerin: differential effects of gestational diabetes mellitus and maternal obesity. Clin Endocrinol. 2013;80:65-72.

43. Henrik S, Birgitta O, Staffan E, Malin LN. Adiponectin, chemerin, cytokines, and dipeptidyl peptidase 4 are released from human adipose tissue in a depot-dependent manner: an in vitro system including human serum albumin. BMC Endocr Disord. 2014;14:7.

44. Pan HY, Guo L, Li Q. Changes of serum omentin-1 levels in normal subjects and in patients with impaired glucose regulation and with newly diagnosed and untreated type 2 diabetes. Diabetes Res Clin Pract. 2010;88:29-33.

45. El-Mesallamy HO, El-Derany MO, Hamdy NM. Serum omentin-1 and chemerin levels are interrelated in patients with type 2 diabetes mellitus with or without ischaemic heart disease. Diabet Med. 2011;28:1194-200.

46. Polkowska A, Szczepaniak I, Bossowski A. Assessment of serum concentrations of ghrelin, Obestatin, Omentin-1, and Apelin in children with type 1 diabetes. Biomed Res Int. 2016;2016:8379294.
47. Souvannavong-Vilivong X, Sitticharoon C, Klinjampa R, Keadkraichaiwat I, Sripong C, Chatree S, Sririwichitchai R, Lertbunnaphong T. Placental expressions and serum levels of adiponectin, visfatin, and omentin in GDM. Acta Diabetol. 2019;56:1121-31.

48. Mousa A, Abell SK, Shorakae S, Harrison CL, Naderpoor N, Hiam D, MorenoAsso A, Stepto NK, Teede HJ, de Courten B. Relationship between vitamin D and gestational diabetes in overweight or obese pregnant women may be mediated by adiponectin. Mol Nutr Food Res. 2017;61.

49. Bawah AT, Seini MM, Abaka-Yawason A, Alidu H, Nanga S. Leptin, resistin and visfatin as useful predictors of gestational diabetes mellitus. Lipids Health Dis. 2019;18:221.

\section{Publisher's Note}

Springer Nature remains neutral with regard to jurisdictional claims in published maps and institutional affiliations.
Ready to submit your research? Choose BMC and benefit from:

- fast, convenient online submission

- thorough peer review by experienced researchers in your field

- rapid publication on acceptance

- support for research data, including large and complex data types

- gold Open Access which fosters wider collaboration and increased citations

- maximum visibility for your research: over $100 \mathrm{M}$ website views per year

At BMC, research is always in progress.

Learn more biomedcentral.com/submissions 